\title{
The Swiss Prospective Autologous Bone Flap Resorption Study (SPARS): a multicentric approach to quantify the complication rate after reimplantation of autologous cryoconserved bone flaps
}

\author{
Lennart Henning Stieglitz ${ }^{1}$
}

Received: 22 March 2015 / Accepted: 23 March 2015 /Published online: 12 April 2015

(C) Springer-Verlag Wien 2015

Dear Editor,

With great pleasure I read the letter by Nicolas Desse et al. entitled "Cranioplasty after decompressive craniectomy: about bone flap preservation." The authors report yet unpublished data from their own department, which do not completely go along with the results presented by our own workgroup in the February 2015 issue of the journal [1]. In a series of 65 patients operated between January 2010 and December 2013, they find major bone flap resorption in only two cases $(7.1 \%)$. They discussed that these two belong to a well-known high-risk group for bone flap resorption being young (18 and 33 years old) and undergoing removal of a very large bone flap that was stored and reimplanted in segments.

The difference in our observations stresses the point that there is need for a prospective multicentric study that analyzes the outcome and complications after reimplantation of cryoconserved bone flaps. In that point there is agreement between Desse et al. and ourselves.

For that reason, we started a prospective multicentric study in January 2015 in Switzerland, to which nearly all of the large and medium-sized Swiss centers agreed to contribute. The study is registered in clinicaltrials.gov under the identifier NCT02320955 and is named "Swiss Prospective Autologous bone flap Resorption Study (SPARS)".
We intend to include 125 patients who underwent reimplantation of their autologous cryoconserved bone flap after decompressive craniectomy. In addition to routine follow-up, they will receive native CT scans 12 and 24 months after reimplantation. Primary endpoint is the incidence of a relevant resorption after 12 months, measured subjectively using the bone flap resorption score we proposed in "What happens to the bone flap? Long-term outcome after reimplantation of cryoconserved bone flaps in a consecutive series of 92 patients" [1] and objectively by volumetric subtraction of the 12- and 24-month postoperative CT scans from the early postoperative one.

Secondary endpoints will be parameters of storage, cryoconservation, fixation, and others.

We hope to finish patient inclusion by the end of 2016 and be able to report the results after finishing the last follow-up in 2018/2019.

Conflicts of interest None.

\section{References}

1. Stieglitz LH, Fung C, Murek M, Fichtner J, Raabe A, Beck J (2014) What happens to the bone flap? Long-term outcome after reimplantation of cryoconserved bone flaps in a consecutive series of 92 patients. Acta Neurochir (Wien). doi:10.1007/s00701-014-2310-7

Lennart Henning Stieglitz

lennart.stieglitz@usz.ch

1 Department of Neurosurgery, Zurich University Hospital, Frauenklinikstrasse 10, 8091 Zurich, Switzerland 